\title{
Primary cardiac malignant fibrous histiocytoma with vulvar metastases: A case report
}

\author{
JUNJIE SUN, RUIHUA LIU, WEIWEI WANG, MINGHUI SUN, \\ LIHONG WANG, XIAOFEI WANG and LEI SHI
}

Department of Ultrasound, Yantai Yuhuangding Hospital, Yantai, Shandong 264000, P.R. China

Received October 13, 2014; Accepted July 30, 2015

DOI: $10.3892 / 01.2015 .3683$

\begin{abstract}
Malignant fibrous histiocytoma (MFH) occurring in the heart is rare. To the best of our knowledge, the current study was the first to report a case of left atrial MFH metastasizing to the vulva. A 37-year-old female presenting with a history of exertional dyspnea underwent echocardiography, which revealed a mass in the left atrium, with a short-wide stalk attached to the posterior wall, extending into the pericardium. Furthermore, the mass resulted in severe mitral stenosis during diastole. The tumor was excised together with a section of the left atrial wall. The initial diagnosis was a left atrial myxoma. However, post-operative frozen section histopathology results indicated a diagnosis of MFH. Following surgery, the patient received chemotherapy, however, vulvar metastases and left pleural effusions were identified after 1 month. Following surgical excision of the vulvar tumor and left thoracentesis, the patient began adjuvant chemotherapy. However, the patient succumbed due to local recurrence 6 months later.
\end{abstract}

\section{Introduction}

Cardiac tumors are divided into primary and secondary tumors. Primary cardiac tumors are rare with a global incidence of $0.0017-0.019 \%(1,2)$. Approximately $75 \%$ of primary cardiac tumors are benign, while $\sim 25 \%$ are malignant (3). Among malignant cardiac sarcomas, angiosarcoma is the most frequent type and primary malignant fibrous histiocytoma (MFH) is the second most common type, with an incidence of $3 \%$ (4). Cardiac sarcomas are often asymptomatic until the tumor causes an obstruction to blood flow and/or metastasizes to distant tissues (5). Therefore, the symptoms of affected patients depends on the location, size and degree of invasiveness of the tumor of the heart, and any distant metastasis (5).

Correspondence to: Professor Lei Shi, Department of Ultrasound, Yantai Yuhuangding Hospital, 20 Yuhuangding East Road, Yantai, Shandong 264000, P.R. China

E-mail: shileiyhdhospital@163.com

Key words: cardiac sarcoma, malignant fibrous histiocytoma of the heart, echocardiography, vulvar sarcoma
The majority of cardiac tumors are identified by transthoracic and/or transesophageal echocardiography (6). Computed tomography (CT) and magnetic resonance imaging (MRI) are also important to establish the extent of invasion and metastases (7). Cardiac tumors may occur at any position of the heart, however, primary MFH of the heart is typically found in the left atrium $(1,3)$.

$\mathrm{MFH}$ is an undifferentiated sarcoma composed of pleomorphic spindle and epithelioid cells with a small number of multinucleated cells. MFH is significantly more invasive than a benign tumor, and recurrence and distant metastasis are common, often leading to an extremely poor prognosis $(8,9)$. Surgical resection is the standard treatment for MFH patients, and ideal resection involves removal of the whole tumor and a section of the normal cardiac tissue (10). However, multimodality treatment including radiotherapy and adjuvant chemotherapy may achieve a better outcome (11). Mean survival for the majority of cases of cardiac sarcoma is 9-11 months (12). A recent study revealed that the mean survival time of MFH patients following resection (incomplete or complete) was $22.2 \pm 6.1$ months (range, 0.6-36.0 months) with 1- and 2-year survival rates of 82.5 and $41.3 \%$, respectively (12). In the current study, the case of a patient exhibiting a giant, rarely observed MFH of the heart with vulvar metastases is presented. Written informed consent was obtained from the patient's family.

\section{Case report}

In March 2009, a 37-year-old female presented to the Yantai Yuhuangding Hospital (Yantai, Shandong, China) with a 3 -month history of progressively increasing exertional dyspnea that was relieved following rest, and a 2-day history of worsening dyspnea. Physical examination identified a blood pressure of $140 / 80 \mathrm{mmHg}$ (normal range, 100-120/60-80 $\mathrm{mmHg}$ ), a body temperature of $37.0^{\circ} \mathrm{C}$ (normal range, $36.2-37.2^{\circ} \mathrm{C}$ ) and a heart rate of 99 beats per minute (bpm; normal range, 60-100 bpm) with a regular rhythm; however, a diastolic murmur was observed upon cardiac auscultation. Subsequent echocardiography revealed a $5.0 \times 2.3-\mathrm{cm}$ mobile mass in the left atrium with a short-wide stalk attached to the posterior wall (Fig. 1A), widely attached to the anterior mitral valve and with a small pericardial effusion. During diastole, the mass protruded through the mitral valve resulting in functional 

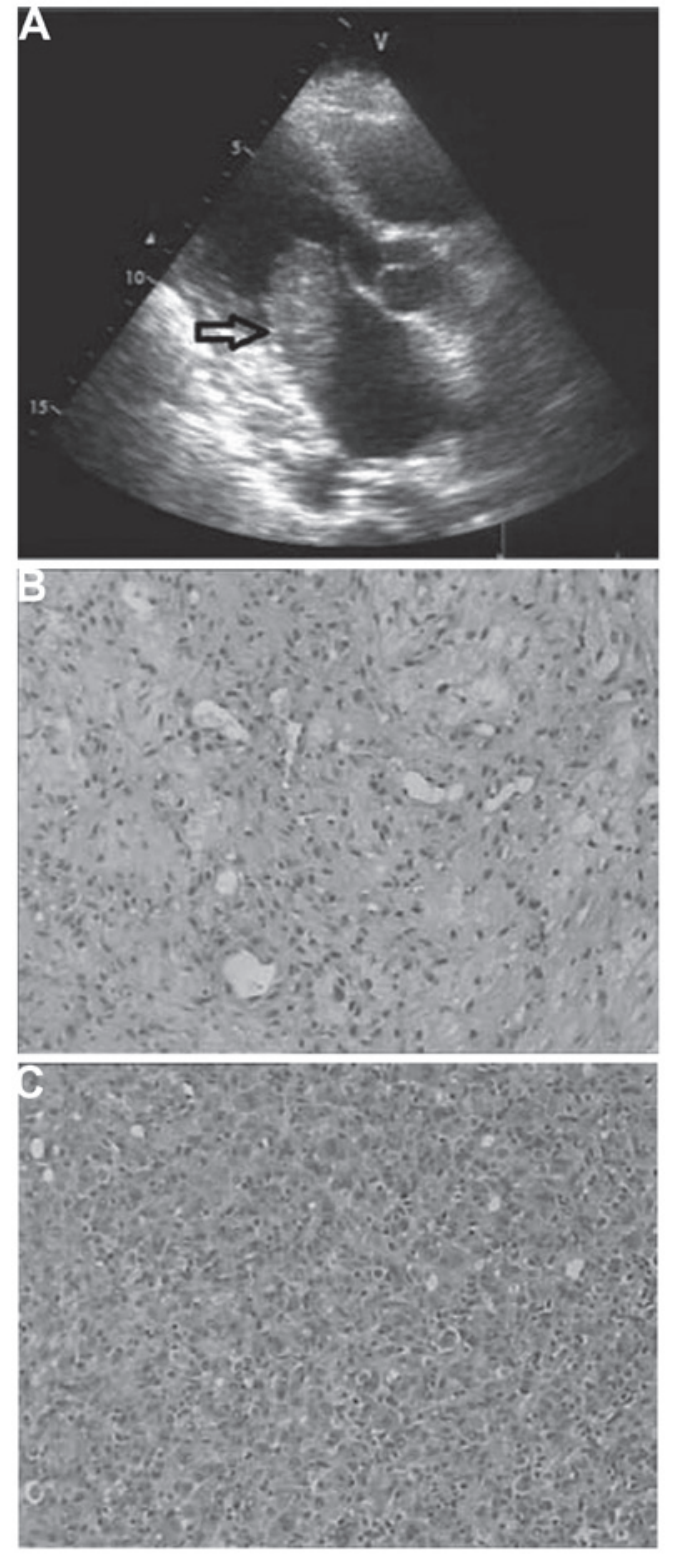

Figure 1. (A) Parasternal long-axis echocardiography view of the tumor (arrow). (B) Light microscopy revealing pleomorphic spindle and epithelioid cells (stain, hematoxylin and eosin; magnification, x100). (C) Immunohistochemistry demonstrating positivty for cluster of differentiation (CD)99 and vimentin, and partial positivity for CD68 (stain, hematoxylin and eosin; magnification, x400). The cells were immunonegative for cytokeratin, epithelial membrane antigen, smooth muscle actin, actin, CD31 and CD34.

mitral stenosis with a peak gradient of $38 \mathrm{mmHg}$ (normal range, $<30 \mathrm{mmHg}$ ). Various auxiliary examinations, including abdomen and brain CT and chest X-rays, showed no signs of tumor in other organs. The initial diagnosis was a left atrial myxoma. Upon pre-operative echocardiography, the left ventricle ejection fraction and pulmonary artery systolic pressure were $63 \%$ (normal range, 50-75\%) and $57 \mathrm{mmHg}$ (normal range, $<30 \mathrm{mmHg}$ ), respectively.

The gold standard therapy for atrial myxoma is complete surgical removal of the tumor (10). In the present case, open-heart surgery with access via a median sternotomy and cardiopulmonary bypass was performed under cardioplegic arrest. At the incision site of the pericardium, the pericar-
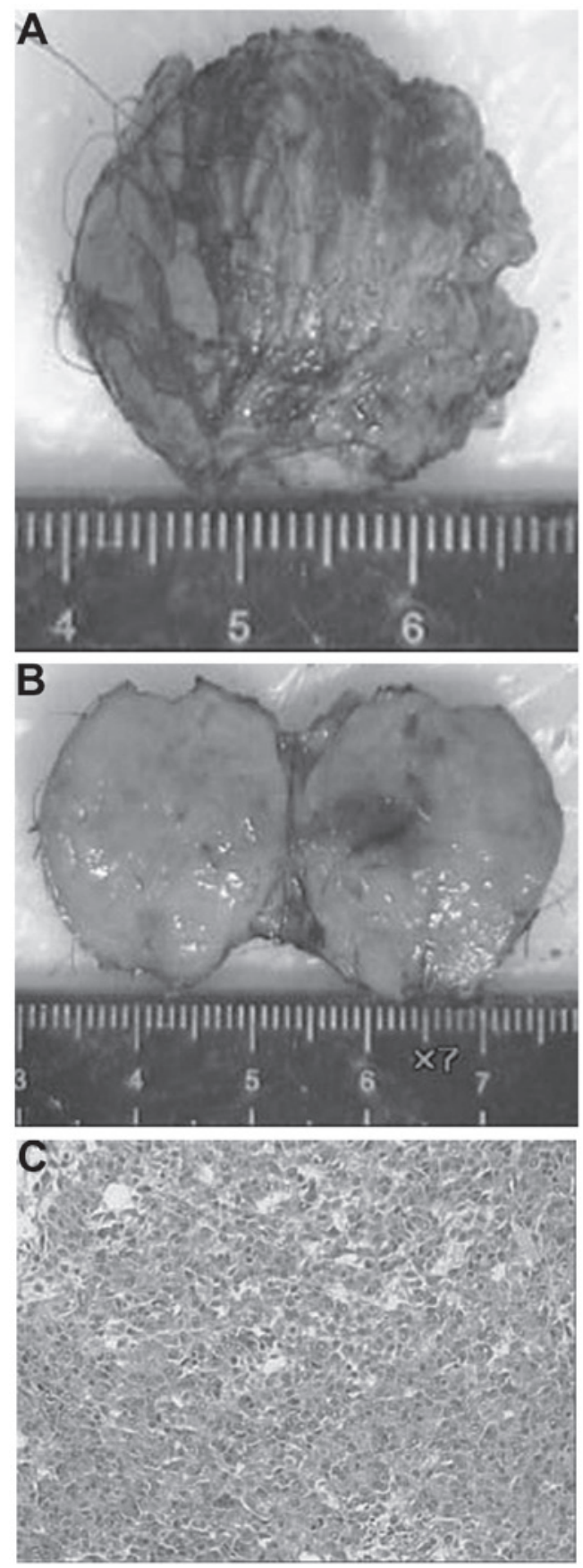

Figure 2. (A) Completely resected vulvar tumor. (B) Whitish yellow hemorrhagic cut surface of the vulvar tumor. (C) Histopathology of the vulvar tumor revealing metastasis of malignant fibrous histiocytoma (stain, hematoxylin and eosin; magnification, x300).

dial effusion was yellowish and clear. The mass appeared grey-white in color and crisp in texture, with a short-wide pedicle attached to the posterior wall, extending into the pericardium. Frozen section pathological examination revealed a diagnosis of MFH; the tumor was heterogenous, composed of spindle and epithelioid cells, interspersed with giant cells. Surgery achieved a complete resection of the tumor of the left atrium, as well as a partial resection of the left atrium adhering to the tumor pedicle and peripheral adipose tissue.

Upon light microscopy (Fig. 1B), a malignant mesenchymal tumor with areas of necrosis was identified. The tumor was composed of highly pleomorphic spindle and 
epithelioid cells, with a small number of multinucleated cells also identified. Immunohistochemistry (Fig. 1C) was positive for cluster of differentiation (CD)99 and vimentin, and partially positive for CD68. The cells were immunonegative for cytokeratin, epithelial membrane antigen, smooth muscle actin, actin, CD31 and CD34. On the basis of the aforementioned histomorphological and immunohistochemical findings, a diagnosis of MFH was formed.

Following cardiac surgery, the patient began six three-weekcycles of chemotherapy $\left(1.2 \mathrm{mg} / \mathrm{m}^{2}\right.$ ifosfamide, day $1 ; 60 \mathrm{mg} / \mathrm{m}^{2}$ epidoxorubicin, day 1) at the Department of Oncology; however, after 1 month, the patient noticed a mass on the left side of the vulva, with no pain. The mass had no capsule (Fig. 2A and B) and was resected under local anesthesia. Histopathology indicated a diagnosis of metastatic MFH (Fig. 2C). A chest X-ray performed after vulval surgery revealed left-sided pleural effusion. Following surgical excision of the vulvar tumor and a left thoracentesis, the patient immediately began four three-weekly cycles of chemotherapy (120 mg paclitaxel, day $1 ; 1.6 \mathrm{~g} / \mathrm{m}^{2}$ gemcitabine, day 1) at the Department of Oncology. However, the combination of surgery and chemotherapy proved to be ineffective, and the patient succumbed due to local recurrence 6 months later.

\section{Discussion}

Primary cardiac malignant neoplasmare rare tumors that account for $25 \%$ of all primary cardiac tumors (1-3). The prevalence of primary cardiac sarcomas was found to be $0.0017 \%$ at autopsy in a large study of six hospitals in the USA, conducted by the American Medical Association (1) and the overall global incidence rate has been recorded as $0.001-0.030 \%(13,14)$. MFH of the heart in particular has an incidence of $3 \%$ of all malignant cardiac sarcomas (4), and typically occurs in the left atrium (3). MFH has high metastatic potential and can spread to various tissues and viscera, including the liver, bones, arms, skeletal muscles, lungs and brain $(11,15-18)$. However, to the best of our knowledge, there have been no previous reports of primary MFH of the heart with vulvar metastases. MFH of the vulva is a rare type of vulvar sarcoma, constituting $<1 \%$ of all vulvar sarcomas $(19,20)$. In 2011, Iwakawa et al reported that there had been nine case reports of MFH of the vulva to date (21) and to the best of our knowledge, no other cases have been reported since.

Echocardiography is a sensitive and non-invasive modality for assessing cardiac tumors, including cardiac $\mathrm{MFH}$; however, it has a low specificity, which may lead to misdiagnosis (6). The initial diagnosis of the current case was left atrial myxoma. The short-wide stalk and the small amount of pericardial effusion observed in the present case may act as an indicator of malignant disease. CT scans and MRI are also effective tools for assessing cardiac tumors, and facilitate the identification of more specific structures of tumors and adjacent invasions (7). The symptoms of patients depend on the location, size and degree of invasiveness of the cardiac tumor. In the current case, the mass protruded through the mitral valve during diastole, resulting in functional mitral stenosis that initiated the onset of dyspnea. The gold standard therapy for cardiac MHF patients without metastases is complete surgical resection of the tumor, with metastasis being the only factor identified to impact survival rate in the literature (22-24).

Ideal resection involves removal of the whole tumor and a portion of the normal cardiac tissue. Mean survival for the majority of cases of cardiac sarcoma is 9-11 months (12). In the current case, complete resection of the left atrial tumor and a partial resection of the left atrial wall and pericardium were performed. However, the tumor metastasized to the vulva 1 month later and the patient succumbed due to local recurrence 13 months after diagnosis. It is hypothesized that adjuvant therapy may be indispensable to prevent local recurrence and distant metastasis, as well as provide improved survival, even in patients that have undergone complete resection (25).

In conclusion, the current study reported a rare case of a cardiac tumor. Echocardiography initially prompted a diagnosis of a left atrial myxoma, however, the post-operative histopathological results determined a diagnosis of $\mathrm{MFH}$, and vulvar metastases were later identified. Multicenter cooperation between cardiac surgeons, cardiologists and oncologists may lead to the identification of an optimal treatment strategy for cardiac MFH, which would improve patient quality of life and increase patient survival rates.

\section{References}

1. Straus R and Merliss R: Primary tumor of the heart. Arch Pathol 39: 74-78, 1945.

2. Neragi-Miandoab S, Kim J and Vlahakes GJ: Malignant tumours of the heart: A review of tumour type, diagnosis and therapy. Clin Oncol (R Coll Radiol) 19: 748-756, 2007.

3. Burke AP, Cowan D and Virmani R: Primary sarcomas of the heart. Cancer 69: 387-395, 1992.

4. Stevens CW, Sears-Rogan P, Bitterman P and Torrisi J: Treatment of malignant fibrous histiocytoma of the heart. Cancer 69: 956-961, 1992.

5. Okby NT and Travis WD: Liposarcoma of the pleural cavity: Clinical and pathologic features of 4 cases with a review of the literature. Arch Pathol Lab Med 124: 699-703, 2000.

6. Grebenc ML, Rosadode Christenson ML,Burke AP, et al: Primary cardiac and pericardial neoplasms: Radiologic-pathologic correlation. Radiographics 20: 1073-1103, 2000.

7. Levine RA, Weyman AE, Dinsmore RE, et al: Noninvasive tissue characterization: Diagnosis of lipomatous hypertrophy of the atrial septum by nuclear magnetic resonance imaging. J Am Coll Cardiol 7: 688-692, 1986.

8. Fletcher CD, Gustafson P, Rydholm A, Willén H and Akerman M: Clinicopathologic re-evaluation of 100 malignant fibrous histiocytomas: Prognostic relevance of subclassification. J Clin Oncol 19: 3045-3050, 2001.

9. Burke A, Jeudy J Jr, Virmani R, Topol EJ and Califf RM (eds). Cardiac tumours. In: Textbook of Cardiovascular Medicine. 3rd edition. Lippincott Williams \& Wilkins, Philadelphia, p710, 2007.

10. Odim J, Reehal V, Laks H, et al: Surgical pathology of cardiac tumors. Two decades at an urban institution. Cardiovasc Pathol 12: 267-270, 2003.

11. Oh SJ, Yeom SY and Kim KH: Clinical implication of surgical resection for the rare cardiac tumors involving heart and great vessels. J Korean Med Sci 28: 717-724, 2013.

12. Bakaeen FG, Reardon MJ, Coselli JS, Miller CC, Howell JF, Lawrie GM, Espada R, Ramchandani MK, Noon GP, Weilbaecher DG and DeBakey ME: Surgical outcome in 85 patients with primary cardiac tumors. Am J Surg 186: 641-647, 2003.

13. McAllister $\mathrm{H}$ and Fenoglio J: Tumors of the cardiovascular system. In: Atlas of Tumor Pathology. 2nd Series. Fascicle 15. American Registry of Pathology/Armed Forces Institute of Pathology, Washington, D.C., pp1-3, 1978.

14. Yu K, Liu Y, Wang H, Hu S and Long C: Epidemiological and pathological characteristics of cardiac tumors: A clinical study of 242 cases. Interact Cardiovasc Thorac Surg 6: 636-639, 2007. 
15. Hsieh SC, Chen CY and Chan WP: Malignant fibrous histiocytoma of the heart: A case report. Acta Cardiol 65: 85-87, 2010.

16. Sheikh AA, Ahmad M, Lone AR and Banday MA: Cardiac metastasis in malignant fibrous histiocytoma. Saudi Med J 29: 1041-1043, 2008.

17. Milicic D, Juretic A, Bulum J, Saric N, Bisof V, Jelic I and Jelasic D: Primary malignant fibrous histiocytoma of the heart with skeletal muscles metastases. J Card Surg 22: 513-516, 2007.

18. Pimentel J, Fernandes AC, Silva R, Ferro J and Cattoni B: Brain metastases of a malignant fibrous histiocytoma presenting as an acute cerebral hemorrhage. Clin Neuropathol 20: 64-69, 2001.

19. Grisaru D, Peyser MR, Bernstein-Lipschitz L, Yaron Y and Lessing JB: Malignant fibrous histiocytoma with myofibroblastic differentiation of the vulva in an adolescent female. Eur J Obstet Gynecol Reprod Biol 79: 219-221, 1998.

20. Taylor RN, Bottles K, Miller TR, and Braga CA: Malignant fibrous histiocytoma of the vulva. Obstet Gynecol 66: 145-148, 1985 .
21. Iwakawa T, Tsuji T, Hamada T, Kamio M, Matsuo T, Yoshinaga M, Kitajima S and Douchi T: Pleomorphic type of malignant fibrous histiocytoma with myxoid stroma of the vulva in a young woman. J Obstet Gynaecol Res 37: 1474-1477, 2011.

22. Park BJ, Bacchetta M, Bains MS, Downey RJ, Flores R, Rusch VW and Girardi LN: Surgical management of thoracic malignancies invading the heart or great vessels. Ann Thorac Surg 78: 1024-1030, 2004.

23. Eckstein R, Gössner W and Rienmüller R: Primary malignant fibrous histiocytoma of the left atrium. Surgical and chemotherapeutic management. Br Heart J 52: 354-357, 1984.

24. Kim MP, Correa AM, Blackmon S, Quiroga-Garza G, Weilbaecher D, Bruckner B, Ramlawi B, Rice DC, Vaporciyan AA and Reardon MJ: Outcomes after right-side heart sarcoma resection. Ann Thorac Surg 91: 770-776, 2011.

25. Reardon MJ, Walkes JC and Benjamin R: Therapy insight: Malignant primary cardiac tumors. Nat Clin Pract Cardiovasc Med 3: 548-553, 2006. 